\title{
Insights into Hepatitis C Transmission in Young Persons who Inject Drugs: \\ Results From a Dynamic Modeling Approach Informed by State-Level Public Health Surveillance Data
}

Authors: Rachel E Gicquelais, Betsy Foxman, Joseph Coyle, Marisa C Eisenberg

\author{
Author Affiliations
}

Department of Epidemiology, University of Michigan School of Public Health, Department of Epidemiology, Ann Arbor, Michigan (Rachel E. Gicquelais, Betsy

Foxman, Marisa C. Eisenberg); Michigan Department of Health and Human Services (Joseph R. Coyle).

\section{Abbreviations}

DAA: Directly acting antiviral

HCV: Hepatitis C Virus

IDU: Injection Drug Use

PWID: People who Inject Drugs or Person who Injects Drugs

US: United States 


\section{Abstract}

Rising use of heroin and prescription opioids are major contributors to increases in Hepatitis C Virus (HCV) incidence in US young adults since the late 1990s. How best to interrupt transmission and decrease HCV prevalence in young persons who inject drugs (PWID) is uncertain, but modeling studies in older populations support interventions that increase HCV treatment among all PWID. We developed a transmission model of young (aged 15-30 years) PWID, which we fit to state-level US HCV surveillance data, and simulated the potential impact of primary (reducing injection initiation), secondary (increasing cessation, reducing injection partners, or reducing injection drug use relapse), and tertiary (HCV treatment) interventions on incident and prevalent $\mathrm{HCV}$ cases. Interventions with primary prevention initiatives (reducing injection initiation) yielded concurrent reductions to HCV incidence and prevalence. Treatment of former PWID led to prevalence reductions but did not reduce incidence. Treatment of current and former PWID without other interventions led to incidence reductions in scenarios with high injection initiation rates, high syringe sharing, and low relapse rates after injection cessation. While these results are specific to Michigan, our approach could be applied in other states conducting HCV surveillance to identify local-level intervention opportunities.

\section{Keywords}

Hepatitis C Virus, Persons who Inject Drugs, Systems Modeling 


\section{INTRODUCTION}

The epidemiology of hepatitis C virus (HCV) in the United States (US) has changed dramatically over the last decade. Increases in HCV incidence among young persons aged approximately 15-30 years have been linked to increases in opioid and injection drug use (IDU), which have been especially large in rural communities (1-5). In the US, up to $2.6 \%$ of adults have injected drugs in their lifetime and $38-48 \%$ of US persons who inject drugs (PWID) have HCV infection, although the prevalence among young PWID is not generally well-characterized (6-10). After decades of chronic infection, HCV leads to liver-associated morbidity and mortality (e.g. cirrhosis and hepatocellular carcinoma) $(11,12)$.

In the US, transmission modeling studies have shaped HCV screening, treatment, and prevention policies by increasing understanding of $\mathrm{HCV}$ transmission dynamics, forecasting prevalence of HCV-related liver diseases, and simulating the impact, costs, and benefits of highly effective directly acting antiviral (DAA) treatments among PWID and other groups disparately burdened by HCV $(11,13-18)$. Several different modeling approaches support the cost-effectiveness of treating PWID with DAAs to interrupt HCV transmission, a strategy known as "treatment as prevention" $(14,15,17,19-42)$. One of the few modeling studies focused on young US PWID ( $<30$ years) showed that, in Chicago, a treatment rate of just 5 per 1,000 young PWID could halve HCV prevalence over 10 years in this age group (from $10 \%$ to $5 \%$ ) (14). While available for Chicago, estimates of HCV prevalence specific to young PWID are not routinely available at the state or local-level throughout the US, limiting the ability to forecast prevalence and 
evaluate the impact of ongoing interventions. We demonstrate here that routine $\mathrm{HCV}$ public health surveillance data collected as part of nationally notifiable and statereportable condition surveillance might be used for this purpose.

$\mathrm{HCV}$ incidence and prevalence increases in young adults and adolescents were first identified using HCV public health surveillance data $(2,43,44)$. As part of HCV surveillance, laboratories and physicians report positive HCV lab results to state health departments, who apply standard case definitions to stage HCV as acute or chronic $(45,46)$. Underreporting of HCV cases limits use of surveillance data for purposes other than description and outbreak monitoring (2). Klevens et al. recently estimated the magnitude of acute HCV under-reporting at approximately 12.3-16.8 cases per case reported (47). In addition to under-reporting, there is high variability in capacity to collect risk factor or demographic information, trace contacts, and connect persons with $\mathrm{HCV}$ infection to testing and treatment $(2,4)$. These limitations have discouraged use of public health surveillance data for HCV transmission modeling.

We developed an HCV transmission model of young PWID in Michigan fit to HCV surveillance data for the state of Michigan (48) that adjusts for case under-reporting and incorporates parameter uncertainty through Latin hypercube sampling of the parameters, a form of stratified random sampling. Model simulations evaluate interventions in a counterfactual framework, including primary prevention (reduced injection initiation), secondary prevention (behavioral initiatives), and tertiary initiatives (HCV treatment), for reducing HCV prevalence and incidence. An evaluation of model fit 
to data given parameters in the literature is used to identify the consistency of parameter ranges with surveillance data and identify scenarios under which certain interventions may be optimal. This modeling framework could be applied to HCV surveillance data from other states and/or adapted for use in other nationally or statenotifiable conditions.

\section{METHODS}

A detailed discussion of the model structure, parameters, initial conditions, surveillance data, and parameter estimation process is available in the Web Methods supplement. Matlab code for model simulation and R (R Foundation for Statistical Computing, Vienna, Austria) code for figures is freely available at https://github.com/epimath/Hepatitis-C-in-Young-PWID.

\section{Model Structure}

An HCV ordinary differential equation (ODE) transmission model of PWID with preferential age mixing was developed in Matlab R2016a (The MathWorks Inc, Natick, MA). The model consists of 9 states per age group, with age groups of 15-19 years, 2024 years, and 25-30 years (Figure 1). Individuals age through groups and transition through a series of compartments within age classes: uninfected, non-PWID $\left(Z_{i}\right)$, uninfected current or former PWID ( $S_{i}$ or $S_{N i}$, respectively), acutely infected current or former PWID ( $A_{i}$ or $A_{N i}$, respectively), chronically infected current or former PWID $\left(C_{i}\right.$ or $\mathrm{C}_{\mathrm{N} i}$, respectively), and treated current or former PWID ( $\mathrm{T}_{i}$ or $\mathrm{T}_{\mathrm{N} i}$, respectively). 
Individuals acquire HCV by injecting drugs; other transmission modes (e.g. perinatal acquisition, unregulated tattoos, sexual transmission) are not considered. Non-PWID initiate IDU at an estimated injection initiation rate, $\phi_{i}$, calibrated to fit HCV surveillance data from an initial value based on the system of equations at steady state (see Web Methods). Susceptible current PWID acquire new infections at an estimated rate $\beta$ through effective contact (i.e. syringe sharing) with an acutely $\left(A_{j}\right)$ or chronically $\left(C_{j}\right)$ infected current PWID in any age class. Contact rates between individuals in each age class are parametrized by a contact matrix, $\Theta$, adapted from social contact patterns in eight European countries (49) (Web Methods). Individuals in treatment compartments ( $\mathrm{T}_{i}$ and $\mathrm{T}_{\mathrm{Ni}}$ ) do not transmit $\mathrm{HCV}$ (i.e. are assumed not to be sharing syringes). Individuals from any of the current PWID classes can stop injecting drugs and move to their adjacent former PWID class at a cessation rate $\delta$ while former PWID can begin injecting again after a period of injection abstinence and enter the current PWID class at a relapse rate $\kappa$.

\section{Surveillance Data, Parameter Estimation, and Parameter Sampling}

The Michigan Department of Health and Human Services (MDHHS) receives reports of HCV diagnoses from healthcare providers and laboratories and stages cases as acute or chronic using standardized national case definitions $(45,46)$. We obtained the number of newly identified acute and chronic HCV cases per year during 2000-2013 and adjusted for under-reporting to surveillance sources using a correction factor developed by Klevens et al. (47). While this correction factor was developed to scale acute HCV cases, the extension to newly reported chronic HCV is justified in persons aged 15-30 
years. These individuals likely acquired infection relatively recently and chronic HCV is under-diagnosed and under-reported among adolescents and young adults.

To incorporate parameter uncertainty, Latin hypercube sampling with 5,000 simulations was used to draw a stratified random sample of parameter sets across plausible ranges gathered through literature review (see Web Methods). To optimize model fit to data, we estimated four unknown parameters (the transmission rate $[\beta]$ and three age-specific injection initiation rates $\left.\left[\phi_{i}\right]\right)$ in each simulation using weighted least squares assuming normally distributed measurement error with variances proportional to the data. Residual sum of squares values after parameter estimation were used to select the best-fitting $50 \%$ of parameter sets to data. To determine if a certain range appeared more consistent with data, we plotted histograms by quartile of fit to the data to visually examine if fit differed along uniformly sampled parameter ranges. Parameter estimation and simulations were run using fminsearch and the ODE15S solver in Matlab.

\section{Potential Interventions}

For each of the $50 \%$ best-fitting parameter sets (2,500 simulations), the model was resimulated after scaling one or more parameters, which provided counterfactual estimates of expected prevalence and new chronic cases (our measure of incidence) in the presence of interventions. We simulated primary prevention interventions (reduced injection initiation $\left[\left(\phi_{i}\right]\right)$, secondary prevention interventions (decreased syringe sharing $[\Theta]$, decreased IDU relapse $[\kappa]$, and increased cessation $[\delta])$, and tertiary prevention interventions (treatment of former $\left[\gamma_{N}\right]$ and current PWID $\left.\left[\gamma_{P}\right]\right)(50)$. Although parameters 
cannot be singly classified into primary, secondary, and tertiary (e.g. current PWID treatment is tertiary [treatment] and secondary [reduces transmission]), these terms classify interventions by their most immediate roles. Parameters were scaled at $10 \%$, $20 \%$, and $40 \%$. We first simulated the expected impact of each intervention alone. Next, we simulated the expected impact of combinations of interventions, specifically to compare the added benefit of secondary interventions on top of primary versus tertiary approaches. Intervention results are summarized by comparing the expected case counts for incident and prevalent chronic HCV cases at year 14 of simulation compared to no intervention.

Two intervention sensitivity analyses were conducted. First, case counts were examined after adding mortality reduction interventions $\left(\eta_{N}\right.$ and $\left.\eta_{P}\right)$ of $40 \%$, which simulate, for example, real-world initiatives to reduce overdose among current PWID, such as bystander overdose response training. Second, treatment duration was compared at 12 weeks versus 1 year, representing treatment durations of currently used DAAs to interferon-based treatments used before 2012, respectively (12).

\section{RESULTS}

We simulated the potential impact of primary (reducing injection initiation), secondary (increasing cessation, reducing injection partners, or reducing injection drug use relapse), and tertiary (HCV treatment) interventions on incident and prevalent chronic HCV cases among young (aged 15-30 years) PWID. The model fit well to Michigan HCV surveillance data, including for the subset of 2,500 parameter sets used to 
simulate interventions (Web Figure 1). Better-fitting parameter sets that were used in intervention simulations tended towards the lower sampled bounds of current PWID prevalence, cessation, and syringe sharing contacts among 15-19 year olds and towards the higher sampled bounds of lifetime PWID prevalence and several other contact parameters (Web Figure 2). The reporting rate had a slight tendency toward the minimum rate, closer to the estimated rate of acute $\mathrm{HCV}$ detection among PWID cases in Klevens et al. (47). Other parameters fit consistently throughout the sampled range (Web Figure 3).

Among the best-fitting 2,500 simulations, the largest predicted reduction in chronic $\mathrm{HCV}$ prevalence at year 14 of simulation occurred by treating current PWID, although variability in results increased with increasing treatment levels (Figure 2A). Primary prevention interventions (decreased injection initiation) predicted the largest and least variable decreases to new chronic $\mathrm{HCV}$ cases across parameter sets, while secondary measures had modest reductions and/or high variability (Figure 2B). New chronic cases were not generally avoided by treating former PWID. Treatment of only former PWID led to small increases or stagnation in predicted new chronic case counts, likely due to reinfection after treatment. Treatment of current or former PWID in the absence of other interventions predicted reductions in new chronic HCV cases in a subset of parameter sets characterized by high injection initiation rates, high numbers of syringe sharing partners, and low rates of relapse after injection cessation. Increased treatment duration and interventions decreasing mortality among current or former PWID did not impact HCV prevalence or new chronic cases (Web Figure 4). 
When considered in combination, adding secondary interventions alongside primary (decreased injection initiation) or tertiary prevention (former PWID treatment) predicted similar average reductions in HCV prevalence at year 14 (Figure 3). Strategies including primary prevention measures averted new chronic cases in all scenarios, and predicted reductions increased by adding secondary and tertiary measures. By comparison, predicted reductions in new chronic cases after including secondary measures alongside treatment were smaller. Interventions including treatment with secondary interventions also exhibited higher variability in predicted case counts across parameter sets compared to those with primary and secondary interventions.

\section{DISCUSSION}

We used an HCV transmission model among young PWID informed by Michigan HCV surveillance data to evaluate the relative benefits of primary, secondary and tertiary interventions to reduce HCV prevalence and incidence. Simulation results suggested that primary prevention initiatives to decrease injection initiation among adolescents and young adults would be the most effective HCV intervention strategy. Both singly and in combination with secondary prevention measures, such as decreasing relapse, increasing cessation, and decreasing syringe sharing, primary prevention predicted reductions to both prevalence and new chronic cases. Predicted prevalence reductions were nearly comparable in magnitude to treatment interventions, although treatment of currently injecting PWID was predicted to be most effective in reducing HCV prevalence. In line with the concept of 'treatment as prevention', current PWID 
treatment also yielded moderate reductions to new chronic HCV cases at high levels of treatment, particularly if combined with secondary and primary prevention initiatives. In addition to reducing the public health impacts of HCV directly, multi-strategy interventions including behavioral interventions focused on IDU would concurrently reduce the economic costs of $\mathrm{HCV}$, which increase with earlier age of infection, and the economic and societal costs of heroin and other opioid use disorders $(51,52)$.

The majority of parameter sets used to simulate interventions tended towards the lower ranges of current PWID prevalence, cessation, and syringe sharing contacts among 1519 year olds and the upper range of lifetime PWID prevalence. Our finding that primary prevention initiatives have the greatest potential for reducing incidence and prevalence together, therefore, are directly generalizable HCV transmission scenarios exhibiting these characteristics. Interestingly, a small number of parameter sets fitting well to data and used to simulate interventions had high injection initiation rates, high numbers of injection partners, and low rates of relapse after injection cessation. Under these conditions, treatment of both current and former PWID was predicted to be highly effective in reducing chronic HCV prevalence and incidence, even in the absence of primary and secondary interventions. Intervention results under each of these two scenarios could be useful in identifying optimal intervention approaches if behavioral characteristics of PWID in a specific locale are known or measured, such as may be done through case or outbreak investigation. 
In sensitivity analyses, mortality reduction interventions among current and former PWID did not qualitatively change predicted primary, secondary, and tertiary intervention results, and absolute increases in predicted HCV prevalence and new chronic cases in the presence of mortality reduction interventions were exceedingly small. Additionally, in agreement with Echevarria et al., treatment predictions were insensitive to treatment duration (14). These results are important given that both could theoretically result in maintenance of a larger PWID population, who could be reinfected and/or contribute to HCV transmission. Practically, secondary and tertiary HCV interventions are and should continue to be coupled alongside mortality reduction interventions. For example, harm reduction programs provide co-located services that include provision of sterile injecting equipment, bystander overdose response training, bloodborne virus testing, and referral to treatment for HCV, Human Immunodeficiency Virus, and other services, such as mental health counseling, substance use disorder treatment, or opioid maintenance therapy (53). Additionally, decreased treatment duration makes treatment more feasible, accessible, and less costly.

Like all modeling exercises, we made several simplifying assumptions, and we were limited by existing data. Our model only considers HCV acquisition through IDU among young PWID and results are not generalizable to other age or risk groups. Cases with missing risk factor data were assumed to be PWID, consistent with PWID being the most common risk factor for $\mathrm{HCV}(3,4,54)$. Because surveillance data suffers from under-reporting and missing data, we applied a reporting rate developed for acute cases to adjust for under-reporting of new chronic cases because there were very few 
acute cases reported during the time period under study (47). To address uncertainty in model parameter values, including the reporting rate, we sampled parameters and used values most consistently fitting to surveillance data to simulate interventions.

Our model assumed homogeneous mixing beyond age and used a contact matrix from Mossong et al., a European study of social contacts, that may not reflect the age-related patterns underlying syringe sharing (49). Mossong et al. provided relevant age-group contacts and because we included sampled parameters, we likely capture many plausible syringe sharing scenarios and present summaries of fit to data to show which scenarios were most consistent with observed data (Web Figure 2). Treatment interventions assumed a wholly treatment-naïve population, however this is likely realistic given the historically low treatment rates among PWID during the time period under study (55-58). Further, treatment was simulated as $100 \%$ effective and should therefore be interpreted as the maximum possible treatment effect, given that randomized controlled trials support that approximately $89 \%$ of patients are cured of HCV by 12 weeks of DAA therapy (59).

\section{Conclusions}

$\mathrm{HCV}$ surveillance data is a valuable data source for understanding HCV transmission and identifying local intervention opportunities among young PWID. In Michigan, primary prevention interventions to decrease injection initiation should be a priority to ensure that HCV prevalence does not further increase among young persons. Treatment for current PWID reduces prevalence more than treating former PWID, thus 
persons at all stages of use or recovery should be connected to HCV treatment alongside primary and secondary prevention interventions.

\section{ACKNOWLEDGMENTS}

Financial support: This work was supported by the Thomas J Francis, Jr. award from the Department of Epidemiology at the University of Michigan School of Public Health and by the Integrated Training in Microbial Systems fellowship at the University of Michigan.

The authors thank Geoff Brousseau, MPH and Kathryn E. Macomber, MPH at the Michigan Department of Health and Human Services for their help in obtaining the surveillance data used in this work.

Conflict of Interest

None declared.

\section{REFERENCES}

1. Tempalski B, Pouget ER, Cleland CM, et al. Trends in the Population Prevalence of People Who Inject Drugs in US Metropolitan Areas 1992-2007. PLOS ONE. 2013;8(6):e64789.

2. Koh HK, Valdiserri RO. Hepatitis C Virus Infection in Younger Persons who Inject Drugs. 2013;(http://aids.gov/pdf/hcv-and-young-pwid-consultation-report.pdf)

3. Zibbell JE, lqbal K, Patel RC, et al. Increases in hepatitis $\mathrm{C}$ virus infection related to injection drug use among persons aged $\leq 30$ years - Kentucky, Tennessee, Virginia, and West Virginia, 2006-2012. MMWR Morb. Mortal. Wkly. Rep. 2015;64(17):453458.

4. Centers for Disease Control and Prevention Division of Viral Hepatitis. Viral Hepatitis Surveillance: United States, 2014. Atlanta, GA: 
2016.(https://www.cdc.gov/hepatitis/statistics/2014surveillance/pdfs/2014hepsurveil lancerpt_rev2016-09-26.pdf)

5. Jordan AE, Blackburn NA, Des Jarlais DC, et al. Past-year prevalence of prescription opioid misuse among those 11 to 30 years of age in the United States: A systematic review and meta-analysis. J. Subst. Abuse Treat. 2017;77:31-37.

6. Lansky A, Finlayson T, Johnson C, et al. Estimating the Number of Persons Who Inject Drugs in the United States by Meta-Analysis to Calculate National Rates of HIV and Hepatitis C Virus Infections. PLoS ONE. 2014;9(5):e97596.

7. Armstrong GL, Wasley A, Simard EP, et al. The prevalence of hepatitis C virus infection in the United States, 1999 through 2002. Ann. Intern. Med. 2006;144(10):705-714.

8. Denniston MM, Jiles RB, Drobeniuc J, et al. Chronic Hepatitis C Virus Infection in the United States, National Health and Nutrition Examination Survey 2003 to 2010. Ann. Intern. Med. 2014;160(5):293-300.

9. Edlin BR, Eckhardt BJ, Shu MA, et al. Toward a More Accurate Estimate of the Prevalence of Hepatitis $\mathrm{C}$ in the United States. Hepatol. Baltim. Md. 2015;62(5):1353-1363.

10. Rosenberg ES, Hall EW, Sullivan PS, et al. Estimation of State-Level Prevalence of Hepatitis C Virus Infection, US States and District of Columbia, 2010. Clin. Infect. Dis. Off. Publ. Infect. Dis. Soc. Am. 2017;

11. Smith B, Morgan R, Beckett $G$, et al. Recommendations for the Identification of Chronic Hepatitis C Virus Infection Among Persons Born During 1945 - 1965. MMWR Recomm Rep. 2012;61(RR-4):1-32.

12. American Association for the Study of Liver Diseases (AASLD), Infectious Diseases Society of America (IDSA). Recommendations for Testing, Managing, and Treating Hepatitis C. 2016 (Accessed October 30, 2016).(http://hcvguidelines.org/sites/default/files/HCVGuidance_October_2016_a.pdf).(Accessed October 30, 2016)

13. Moyer VA, U.S. Preventive Services Task Force. Screening for hepatitis C virus infection in adults: u.s. Preventive services task force recommendation statement. Ann. Intern. Med. 2013;159(5):349-357.

14. Echevarria D, Gutfraind A, Boodram B, et al. Mathematical Modeling of Hepatitis C Prevalence Reduction with Antiviral Treatment Scale-Up in Persons Who Inject Drugs in Metropolitan Chicago. PloS One. 2015;10(8):e0135901.

15. Hickman $M$, De Angelis $D$, Vickerman $P$, et al. Hepatitis $C$ virus treatment as prevention in people who inject drugs: testing the evidence. Curr. Opin. Infect. Dis. 2015;28(6):576-582. 
16. Leidner AJ, Chesson HW, Xu F, et al. Cost-effectiveness of hepatitis $C$ treatment for patients in early stages of liver disease. Hepatol. Baltim. Md. 2015;61(6):18601869.

17. Durham DP, Skrip LA, Bruce RD, et al. The Impact of Enhanced Screening and Treatment on Hepatitis $\mathrm{C}$ in the United States. Clin. Infect. Dis. Off. Publ. Infect. Dis. Soc. Am. 2016;62(3):298-304.

18. Linthicum MT, Gonzalez YS, Mulligan K, et al. Value of expanding HCV screening and treatment policies in the United States. Am. J. Manag. Care. 2016;22(6 Spec No.):SP227-235.

19. Martin NK, Vickerman P, Hickman M. Mathematical modelling of hepatitis C treatment for injecting drug users. J. Theor. Biol. 2011;274(1):58-66.

20. Martin NK, Vickerman P, Foster GR, et al. Can antiviral therapy for hepatitis C reduce the prevalence of $\mathrm{HCV}$ among injecting drug user populations? A modeling analysis of its prevention utility. J. Hepatol. 2011;54(6):1137-1144.

21. Martin NK, Pitcher AB, Vickerman $P$, et al. Optimal control of hepatitis $C$ antiviral treatment programme delivery for prevention amongst a population of injecting drug users. PloS One. 2011;6(8):e22309.

22. Durier N, Nguyen C, White LJ. Treatment of hepatitis C as prevention: a modeling case study in Vietnam. PloS One. 2012;7(4):e34548.

23. Hellard ME, Jenkinson $R$, Higgs $P$, et al. Modelling antiviral treatment to prevent hepatitis $\mathrm{C}$ infection among people who inject drugs in Victoria, Australia. Med. J. Aust. 2012;196(10):638-641.

24. Martin NK, Vickerman $P$, Miners $A$, et al. Cost-effectiveness of hepatitis $C$ virus antiviral treatment for injection drug user populations. Hepatol. Baltim. Md. 2012:55(1):49-57.

25. Martin NK, Vickerman P, Grebely J, et al. Hepatitis C virus treatment for prevention among people who inject drugs: Modeling treatment scale-up in the age of directacting antivirals. Hepatol. Baltim. Md. 2013;58(5):1598-1609.

26. Martin NK, Hickman M, Hutchinson SJ, et al. Combination interventions to prevent HCV transmission among people who inject drugs: modeling the impact of antiviral treatment, needle and syringe programs, and opiate substitution therapy. Clin. Infect. Dis. Off. Publ. Infect. Dis. Soc. Am. 2013;57 Suppl 2:S39-45.

27. de Vos AS, Kretzschmar MEE. Benefits of hepatitis $C$ virus treatment: a balance of preventing onward transmission and re-infection. Math. Biosci. 2014;258:11-18.

28. Bennett $H$, McEwan $P$, Sugrue $D$, et al. Assessing the Long-Term Impact of Treating Hepatitis C Virus (HCV)-Infected People Who Inject Drugs in the UK and 
the Relationship between Treatment Uptake and Efficacy on Future Infections. PloS One. 2015;10(5):e0125846.

29. Innes H, Goldberg D, Dillon J, et al. Strategies for the treatment of Hepatitis $C$ in an era of interferon-free therapies: what public health outcomes do we value most? Gut. 2015;64(11):1800-1809.

30. Lima VD, Rozada I, Grebely J, et al. Are Interferon-Free Direct-Acting Antivirals for the Treatment of HCV Enough to Control the Epidemic among People Who Inject Drugs? PloS One. 2015;10(12):e0143836.

31. Martin NK, Foster GR, Vilar J, et al. HCV treatment rates and sustained viral response among people who inject drugs in seven UK sites: real world results and modelling of treatment impact. J. Viral Hepat. 2015;22(4):399-408.

32. Scott $N$, McBryde E, Vickerman $P$, et al. The role of a hepatitis $C$ virus vaccine: modelling the benefits alongside direct-acting antiviral treatments. BMC Med. 2015;13:198.

33. Ayoub $\mathrm{HH}$, Abu-Raddad LJ. Impact of treatment on hepatitis $\mathrm{C}$ virus transmission and incidence in Egypt: A case for treatment as prevention. J. Viral Hepat. 2016;

34. Bennett H, Gordon J, Jones B, et al. Hepatitis C disease transmission and treatment uptake: impact on the cost-effectiveness of new direct-acting antiviral therapies. Eur. J. Health Econ. HEPAC Health Econ. Prev. Care. 2016;

35. Cousien A, Tran VC, Deuffic-Burban S, et al. Hepatitis $C$ treatment as prevention of viral transmission and liver-related morbidity in persons who inject drugs. Hepatol. Baltim. Md. 2016;63(4):1090-1101.

36. Martin NK, Vickerman P, Brew IF, et al. Is increased HCV case-finding combined with current or 8-12 week DAA therapy cost-effective in UK prisons? A prevention benefit analysis. Hepatol. Baltim. Md. 2016;

37. Rozada I, Coombs D, Lima VD. Conditions for eradicating hepatitis C in people who inject drugs: A fibrosis aware model of hepatitis $C$ virus transmission. J. Theor. Biol. 2016;395:31-39.

38. Scott N, McBryde ES, Thompson A, et al. Treatment scale-up to achieve global HCV incidence and mortality elimination targets: a cost-effectiveness model. Gut. 2016;

39. van Santen DK, de Vos AS, Matser A, et al. Cost-Effectiveness of Hepatitis C Treatment for People Who Inject Drugs and the Impact of the Type of Epidemic; Extrapolating from Amsterdam, the Netherlands. Plos One. 2016;11(10):e0163488. 
40. Cousien $A$, Leclerc $P$, Morissette $C$, et al. The need for treatment scale-up to impact HCV transmission in people who inject drugs in Montréal, Canada: a modelling study. BMC Infect. Dis. 2017;17(1):162.

41. Gountas I, Sypsa V, Anagnostou O, et al. Treatment and primary prevention in people who inject drugs for chronic hepatitis $C$ infection: is elimination possible in a high-prevalence setting? Addict. Abingdon Engl. 2017;

42. Stone J, Martin NK, Hickman M, et al. Modelling the impact of incarceration and prison-based hepatitis $\mathrm{C}$ virus $(\mathrm{HCV})$ treatment on $\mathrm{HCV}$ transmission among people who inject drugs in Scotland. Addict. Abingdon Engl. 2017;

43. Church D, Barton K, Elson F, et al. Notes from the Field: Risk Factors for Hepatitis C Virus Infections Among Young Adults --- Massachusetts, 2010. Morb. Mortal. Wkly. Rep. 2011;60(42):1457-1458.

44. Onofrey S, Church D, Kludt $P$, et al. Hepatitis C Virus Infection Among Adolescents and Young Adults --- Massachusetts, 2002--2009. Morb. Mortal. Wkly. Rep. 2011;60(17):537-541.

45. Hepatitis C, Acute | 2012 Case Definition. (https://wwwn.cdc.gov/nndss/conditions/hepatitis-c-acute/case-definition/2012/). (Accessed October 30, 2016)

46. Hepatitis C, Past or Present | 2012 Case Definition. (https://wwwn.cdc.gov/nndss/conditions/hepatitis-c-chronic/case-definition/2012/). (Accessed October 30, 2016)

47. Klevens RM, Liu S, Roberts $\mathrm{H}$, et al. Estimating acute viral hepatitis infections from nationally reported cases. Am. J. Public Health. 2014;104(3):482-487.

48. Michigan Department of Community Health Viral Hepatitis Surveillance and Prevention Unit. Young Adults with Hepatitis C Summary Report. 2013; (http://www.michigan.gov/documents/mdch/Young_Adult_Summary_Report_ v6_412759_7.pdf)

49. Mossong J, Hens N, Jit M, et al. Social Contacts and Mixing Patterns Relevant to the Spread of Infectious Diseases. PLOS Med. 2008;5(3):e74.

50. National Institute on Drug Abuse. Diagnosis and Treatment of Drug Abuse in Family Practice. (https://archives.drugabuse.gov/diagnosistreatment/diagnosis7.html). (Accessed February 16, 2017)

51. Razavi H, ElKhoury AC, Elbasha E, et al. Chronic hepatitis C virus (HCV) disease burden and cost in the United States. Hepatology. 2013;57(6):2164-2170.

52. Jiang R, Lee I, Lee TA, et al. The societal cost of heroin use disorder in the United States. PLOS ONE. 2017;12(5):e0177323. 
53. United States Department of Health and Human Services (HHS), Office of the Surgeon General. Facing Addiction in America: The Surgeon General's Report on Alcohol, Drugs, and Health. Washington, DC: HHS; 2016.(https://addiction.surgeongeneral.gov/surgeon-generals-report.pdf)

54. Suryaprasad AG, White JZ, Xu F, et al. Emerging Epidemic of Hepatitis C Virus Infections Among Young Nonurban Persons Who Inject Drugs in the United States, 2006-2012. Clin. Infect. Dis. 2014;59(10):1411-1419.

55. Mehta SH, Genberg BL, Astemborski J, et al. Limited Uptake of Hepatitis C Treatment Among Injection Drug Users. J. Community Health. 2008;33(3):126.

56. Grebely J, Genoway KA, Raffa JD, et al. Barriers associated with the treatment of hepatitis C virus infection among illicit drug users. Drug Alcohol Depend. 2008;93(1-2):141-147.

57. Bajis $S$, Dore GJ, Hajarizadeh B, et al. Interventions to enhance testing, linkage to care and treatment uptake for hepatitis $C$ virus infection among people who inject drugs: A systematic review. Int. J. Drug Policy. 2017;

58. Global Hepatitis Report 2017. Geneva: World Health Organization; 2017.(http://apps.who.int/iris/bitstream/10665/255016/1/9789241565455eng.pdf?ua=1)

59. Ferreira VL, Tonin FS, Assis Jarek NA, et al. Efficacy of Interferon-Free Therapies for Chronic Hepatitis C: A Systematic Review of All Randomized Clinical Trials. Clin. Drug Investig. 2017;37(7):635-646. 


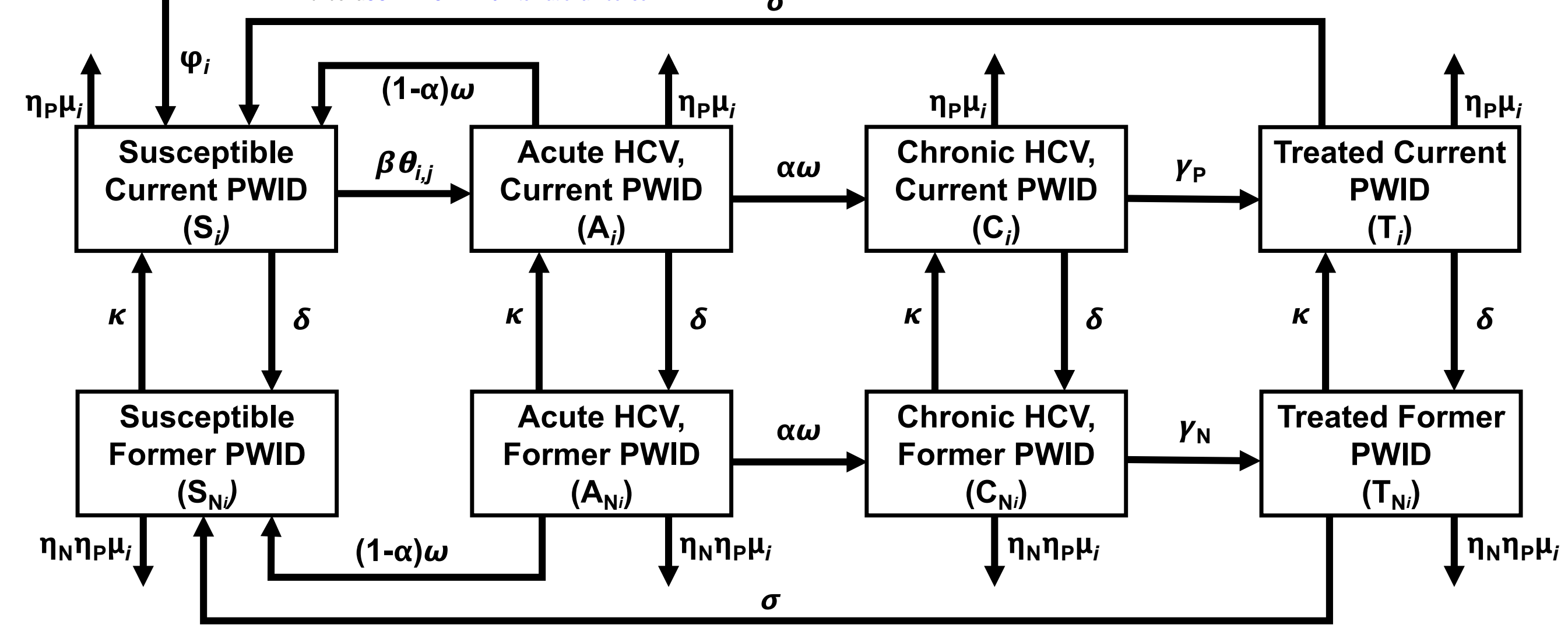

Figure 1. Compartmental Model of HCV Transmission among Young PWID in Michigan

States and parameters controlling flows between compartments of an HCV transmission model among young PWID in Michigan. Non-PWID $\left(Z_{i}\right)$ begin injecting drugs $\left(S_{i}\right)$ at a rate $\phi_{i}$ and acquire acute infection $\left(\mathrm{A}_{i}\right)$ through effective contact with an HCV-infected PWID $\left(\mathrm{A}_{j}\right.$ or $\left.\mathrm{C}_{j}\right)$ of the same or discordant age $\left(\Theta_{i, j}\right)$ at a transmission rate $(\beta)$. Chronic infection $\left(C_{i}\right)$ develops at a rate $\omega$ among a proportion of acute cases, $\alpha$, and resolves in the remaining 1- $\alpha$ acute cases. Chronic infection can be treated $\left(T_{i}\right)$ at a rate $\gamma_{\mathrm{p}}$, for a duration of $\sigma^{-1}$ years, after which point susceptibility to reinfection ensues. PWID stop injecting drugs at a rate $\delta$ and transition to former PWID states (denoted by State $_{\mathrm{Ni}}$ ). Former PWID can begin injecting drugs after a period of abstinence at a rate $\kappa$. Death occurs at a rate $\mu_{i}$, which is elevated among current PWID by a factor $\eta_{P}$. Former PWID mortality rates are less disparate from non-PWID by multiplying the current PWID mortality increase factor by a protective factor $\eta_{\mathrm{N}}$. Subscript $i$ denotes parameter or state age class $(1=15-$ 19 years, $2=20-24$ years, $3=25-30$ years) and subscript $j$ denotes the age group of contacts from whom susceptible current PWID $\left(S_{i}\right)$ can acquire infection. Individuals move through age groups based on the duration predicted by the age range captured in each group $\left(v_{\mathrm{i}}\right.$, not depicted for simplicity) and new 15 year-olds are added to the non-PWID compartment each year at a rate $\left(v_{0} Z_{0}\right.$, not depicted for simplicity). 

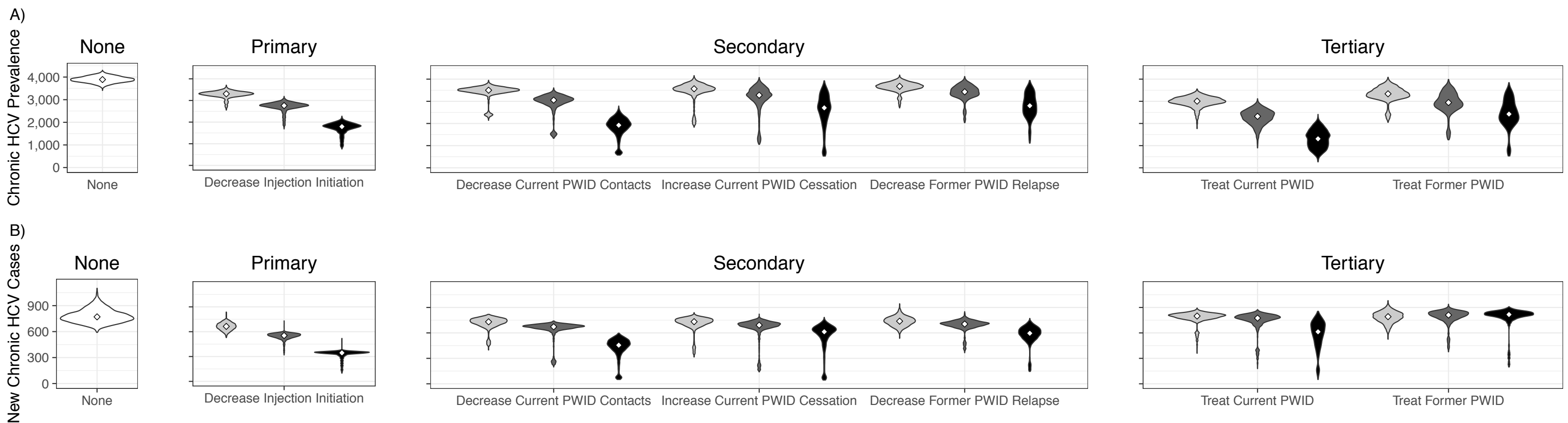

Intervention Level $\square$ None $\square 10 \% \square 20 \% \square 40 \%$

\section{Figure 2. Counterfactual Simulation of Single Interventions}

The distribution of predicted surveillance-detected HCV prevalence (top) and number of new chronic HCV cases (bottom) for each of 6 interventions among the best-fitting 50\% of parameter sets to data are depicted as violin plots. Compared to no intervention (white, right hand side), treating current PWID and decreased injection initiation were associated with the largest predicted reductions to HCV prevalence (A). Reducing injection initiation was associated with the largest predicted reductions to new chronic HCV cases (B). 
A)

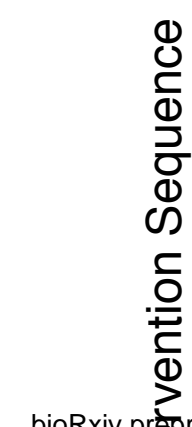

+Treat Current PWID

Decrease Relapse

Increase Cessation

C)

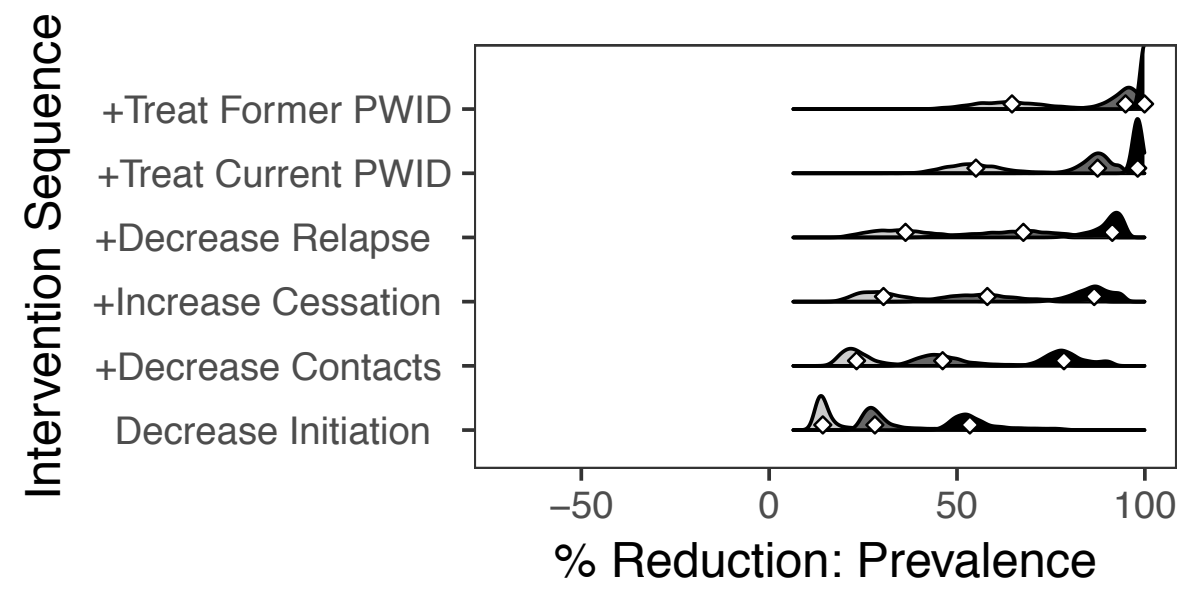

\% Reduction: New Chronic
B)

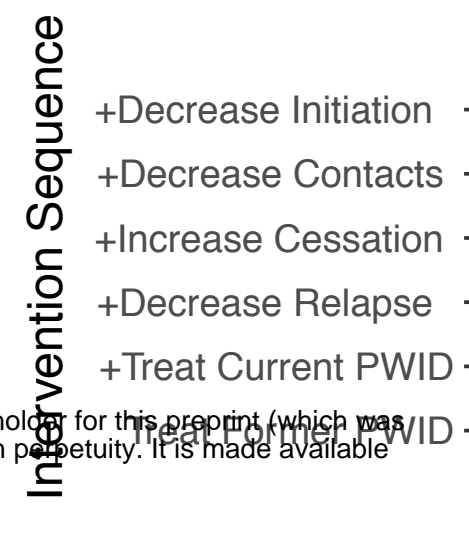

D)

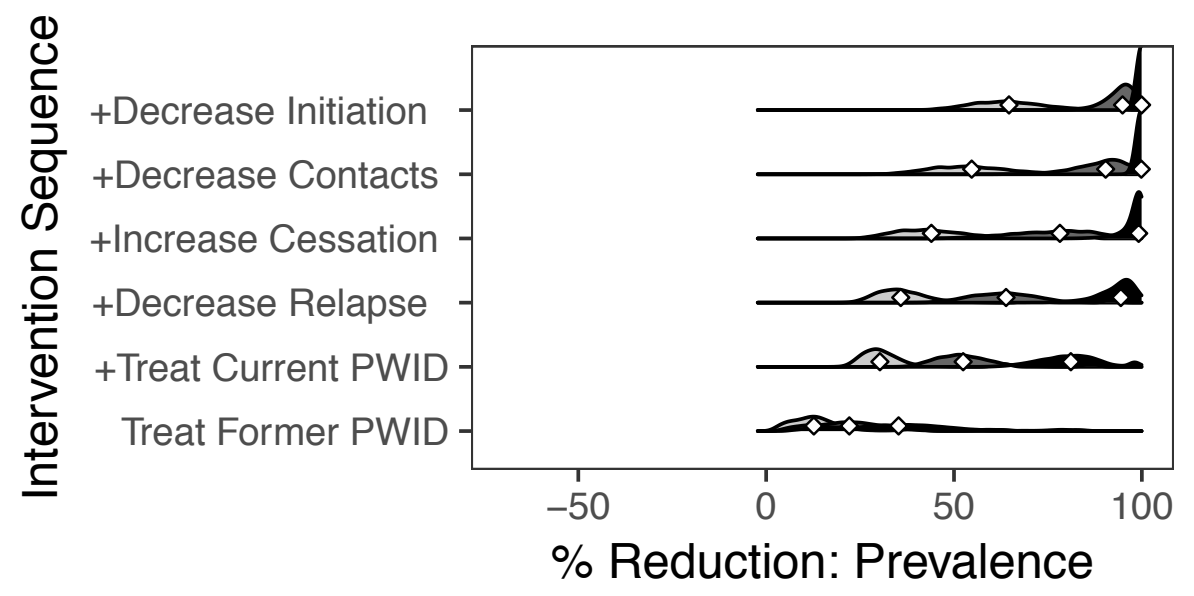

Intervention $\% \square 10 \% \square 20 \% \square 40 \%$

\section{Figure 3. Counterfactual Simulation of Combined Interventions}

Histograms of the predicted percent reduction in new chronic cases (panels $A$ and $B$ ) and prevalence (panels $C$ and D) after 14-years simulation is plotted for each of 2,500 parameter sets. All percent reduction calculations are calculated in comparison to no intervention and interventions are sequentially added from a base of primary (panels $A$ and $C$ ) versus tertiary (panels $B$ and $D$ ) interventions. Diamonds denote the median percent reduction. Primary prevention through decreased initiation of non-PWID to injecting was concurrently associated with predicted reductions to new chronic HCV cases (A) and prevalence (B). Reductions continued with the introduction of secondary and tertiary measures. Tertiary measures (HCV treatment) were associated with predicted reductions to prevalence (D) but not new chronic cases (B). Introduction of secondary on top of tertiary prevention measures predicted decreases in new chronic cases, although predicted declines to new chronic cases were smaller with the addition of additional interventions until all primary, secondary, and tertiary interventions were engaged simultaneously. The variability in predicted reductions in new chronic cases (panels $A$ and $B$ ) was larger than prevalence reductions (panels $C$ and D). Interventions including treatment with secondary interventions (panels B and D) exhibited higher variability in predicted case counts compared to those with primary and secondary interventions (panels $\mathrm{A}$ and $\mathrm{C}$ ). 